\title{
Using Evolutionary Computation Methods to Support Analytical Models for the Evolution and Maintenance of Conditional Strategies in Chthamalus anisopoma
}

\author{
Gloria Childress Townsend \\ DePauw University \\ Computer Science Department \\ Greencastle, IN 46135 \\ 765.658 .4726 \\ gct@depauw.edu
}

\author{
Wade N. Hazel \\ DePauw University \\ Biological Sciences Department \\ Greencastle, IN 46135 \\ 765.658 .4778 \\ wnh@depauw.edu
}

\author{
Rick Smock \\ DePauw University \\ Mathematics Department \\ Greencastle, IN 46135 \\ 765.658 .4729 \\ rcs@depauw.edu
}

\begin{abstract}
Biologists have developed models to explain why different environmentally induced morphs of the same organism exist over time. Such conditional strategies are a common form of adaptation to variable environments, whereby an environmental cue allows some individuals to respond to the cue and develop into a morph that is different from the morph of individuals that do not receive the cue. Recently, these efforts have resulted in two different analytical models that give somewhat different predictions. Here we apply evolutionary computation methods to test the two analytical models. The results bear a remarkable similarity to the results of one of the two analytical models. The paper that follows presents the details of a biological application involving snails and barnacles (that occur naturally in two different morphs), moving then to an explanation of two competing mathematical models of the application. Finally, the interdisciplinary paper, which coordinates three separate research projects of a biologist, a mathematician and a computer scientist, describes the evolutionary computation methods used to support one of the two competing analytical models.
\end{abstract}

\section{Categories and Subject Descriptors \\ Biological Applications}

\section{General Terms}

Algorithms, experimentation

\section{Keywords}

Conditional strategies, threshold model, induced defense, alternative phenotypes, evolutionary computation, evolution strategies

\section{PROJECT OVERVIEW FROM THE BIOLOGICAL PERSPECTIVE}

In this section we illustrate the conditional strategy of predatorinduced defense in the intertidal barnacle Chthamalus anisipoma [7, 8]. A major predator of Chthamalus anisipoma is the gastropod snail Acanthina angelica, which uses a spine on the lip of its shell to break open the opercular valves of the typical conic morph of the adult barnacle, in order to kill the barnacle and consume it.

Figure 1 below (courtesy of W. N. Hazel) demonstrates the predator snail, Acanthina angelica. The arrow points to the spine.

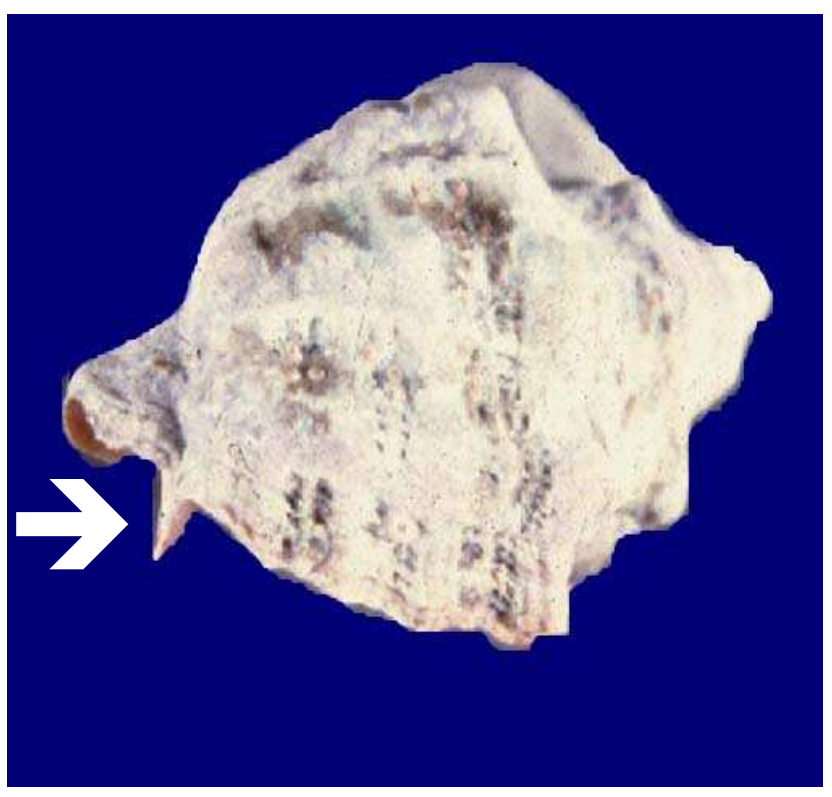

Figure 1: Acanthina angelica, the snail

Permission to make digital or hard copies of all or part of this work for personal or classroom use is granted without fee provided that copies are not made or distributed for profit or commercial advantage and that copies bear this notice and the full citation on the first page. To copy otherwise, or republish, to post on servers or to redistribute to lists, requires prior specific permission and/or a fee.

GECCO'05, June 25-29, 2005, Washington, DC, USA.

Copyright 2005 ACM 1-59593-010-8/05/0006 ..\$5.00. 
Acanthina. The conic morph, while not resistant to attack by Acanthina, has greater reproductive success, if not attacked, than does the bent morph [7]. At least some barnacles are conditional strategists, and develop the bent morph in response to exposure as juveniles to the snail $[8,11]$.

Figure 2 below (also courtesy of W. N. Hazel) contains both conic and bent morphs. The arrows point to bent barnacles.

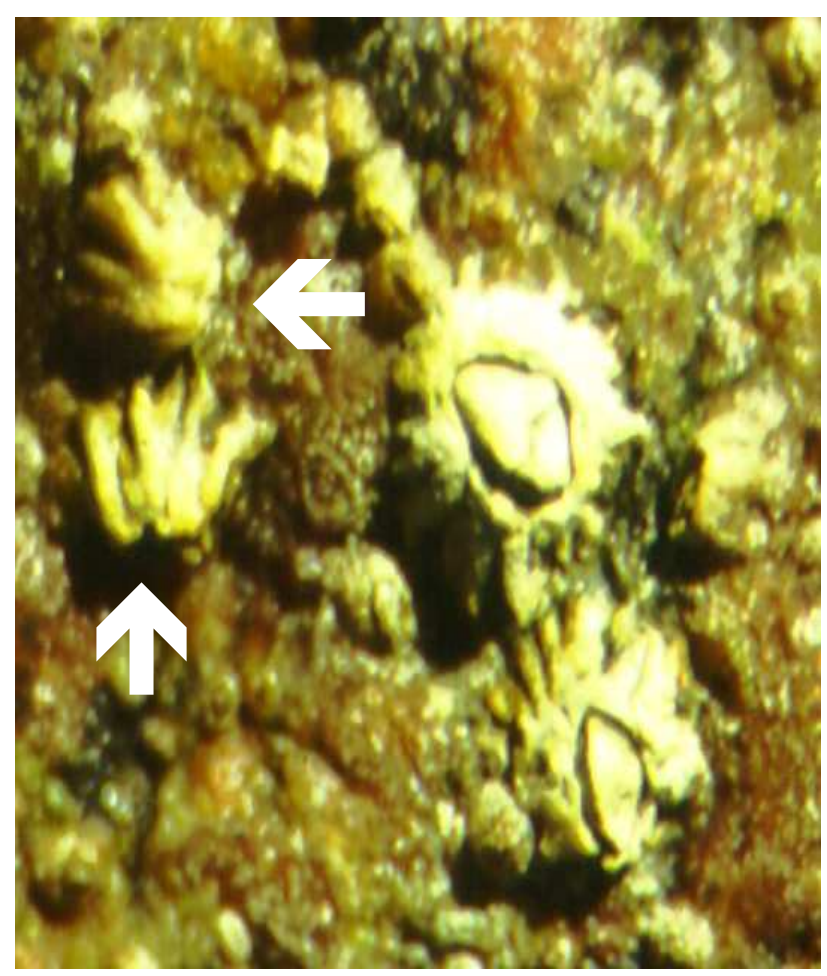

Figure 2: Defended/bent and

Undefended/conic Morphs

Chthamalus occurs throughout the Gulf of California. Because barnacle larvae inhabit open water, we assume that the gulf represents a single panmictic population. Acanthina occurs only in the northern half of the Gulf of California; it is restricted to crevices and boulders that it seeks as refuges.

\subsection{The Strategic Model}

Building on the work of Levins [6], several theoreticians have developed strategic models to investigate the conditions necessary for the evolution and maintenance of conditional strategies [7, 12, 13, 15]. The results of these models have generally been consistent in emphasizing spatial or temporal environmental variation, fitness trade-offs for the alternative tactics of the strategy in different environments, and the reliability of environmental cues as important conditions for the evolution of conditional strategies. (Note that fitness, in the remainder of the paper, refers to biological fitness, not fitness in the evolutionary computation sense of the word.)
Lively's [7, 10] model is based on his work on predator-induced defense described above $[8,9]$, but the model can easily be applied to other conditional strategies. The model involves an evolutionary game between two unconditional strategists, which produce defended and undefended adult morphs, and a conditional strategist, which produces the defended morph if exposed to an environmental cue indicative of the presence of the predator and the undefended morph if not exposed to the cue.

Juveniles receive a cue, if Acanthina crawls over the barnacles, depositing a typical, snail-like trail containing chemicals. If the sensitivity of Chthamalus anisopoma lies within a particular range, then the chemicals induce the juvenile to assume the bent morph as it matures. The adult bent morph then resists the snail, if it resides in the harsh patch.

The defended morph is assumed to be resistant to attack by some predator, while the undefended morph is susceptible to attack. Using game theory, Lively determined under what circumstances the conditional strategy would be favored, when parameter values (described in the following paragraphs) are varied (i.e. the conditional strategy is the evolutionary stable strategy (ESS)).

To examine the effect of environmental variation on the outcome, juveniles are assumed to settle in either of two patches at random. The "benign" patch, in which adults are not attacked by the predator, was entered with probability $\mathrm{p}$, and the "harsh" patch, in which they are ultimately attacked, with probability 1-p.

To examine the effects of variation in cue reliability on the outcome, exposure of juveniles to the cue could imperfectly correlate with attack by the predator. The reliability of the environmental cue in the two patches was governed by the variables $F$ and $G$, where $F$ is the probability that the cue is not present in the benign patch, and $\mathrm{G}$ is the probability that the cue is present in the harsh patch. As such, F and $G$ give the reliability of the cue in the benign and harsh patches, respectively.

The effect of fitness differences in the strategies was modeled by assuming that the undefended morph was unable to survive in the harsh patch. Both morphs were assumed to survive equally well in the benign patch, but development of the defended morph could carry a fitness cost due to reduced fecundity of 1-k; $\mathrm{k}$ is the fitness of defended individuals relative to undefended individuals, which have fitness of 1 in the benign patch and 0 in the harsh patch. If $k$ $=1$, there is no patch dependent fitness tradeoff for the defended morph.

The strategic model is a four-patch model with respect to selection on the three strategies; individuals can be attacked or not, and the cue can be present or not, in all four possible combinations. The results of the strategic model are displayed in Figure 3, which shows the winning strategies, $D$ for defended (bent), $U$ for undefended (conic), and $\mathrm{C}$ for conditional, over the full range of values for $\mathrm{p}$ and $\mathrm{G}$, with $\mathrm{k}=\mathrm{F}=0.5$. Cue reliability, environmental heterogeneity, and the fitnesses of the two morphs interact to determine which strategy, $\mathrm{U}, \mathrm{D}$ or $\mathrm{C}$, is the evolutionary stable strategy (ESS). Notice that there is no ambiguity in figure 3; that is, one of the three strategies (U, D or C) is always the clear winner. 


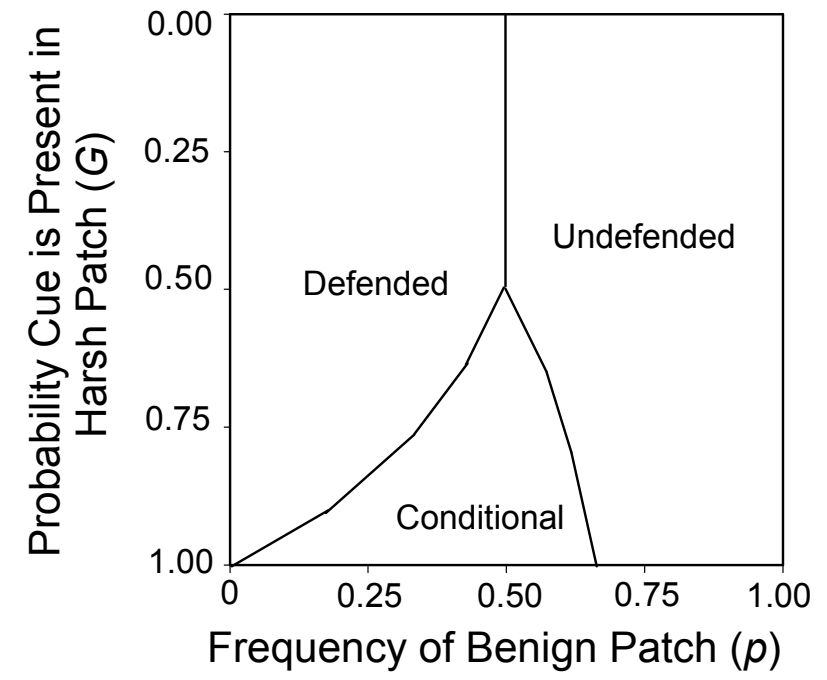

Figure 3: Parameter space for the Strategic Model showing winning strategy, different combinations of patch frequency and cue reliability in the harsh patch.

\subsection{The Environmental Threshold Model (ET)}

Hazel et al. [2,5] used a quantitative genetic model (the Environmental Threshold model or ET) to examine the ecological conditions leading to the evolution and maintenance of conditional strategies $[2,3,5]$. The ET model differs from the ESS model in that the ET postulates genetic differences in sensitivity (response threshold) to the environmental cue that influences which tactic of the conditional strategy is exercised. This difference allows the conditional strategy to be modeled using quantitative genetic theory.

The ET model assumes that normally distributed heritable variation in threshold response, $\mathrm{x}$, [probability density function $\mathrm{f}(\mathrm{x})$ ] to the environmental cue $(\mathrm{t})$ underlies the alternative tactics of the conditional strategy. Threshold is inversely scaled with the value of the cue, such that as threshold increases, the probability of expression of the bent morph also increases. Theoretically, individuals can have response thresholds so low that they develop as bent, even in the absence of exposure to the predator, while others can have thresholds so high that they develop as conic even when the cue is present. As a result, if exposure to the predator as a juvenile is represented as cue value $t_{1}$, and the lack of exposure is represented as $t_{0}$, then only those individuals whose thresholds, $\mathrm{x}$, lie between $\mathrm{t}_{1}$ and $\mathrm{t}_{0}$ will be conditional strategists. Juveniles whose thresholds are greater than $t_{1}$ would always develop as undefended, while those whose thresholds are less than $t_{0}$ would always develop as defended.

\section{THE MATHEMATICAL PERSPECTIVE}

\subsection{Quantitative Genetic Theory}

Introducing variation in cue reliability results in a four different outcomes with respect to selection on threshold, since within the harsh or benign patches, the cue may or may not be present. In each patch, selection on threshold, acting through the fitnesses of the alternative tactics, contributes to the overall selection differential on response threshold, according to the frequency of the patch. Given that variation in response threshold is heritable, selection differential determines response to selection. Selection differentials greater than zero will result in an increase in mean response thresholds between generations. When the selection is zero, then mean threshold will not change between generations. In the patches in which individuals are not attacked, selection favors low thresholds, while in the patches in which individuals are attacked, high thresholds are favored, with selection truncating the distribution of thresholds either at $t_{0}$ for individuals not exposed to the cue, or at $t_{1}$ for individuals exposed to the cue. The general formula for the selection differential, S, taking into account cue reliability is given:

$$
\begin{aligned}
& S(\mu)=\frac{\sigma^{2}}{Q}\left[\left(\alpha_{1}-\beta_{1}\right) q_{1} f\left(t_{0}\right)+\left(\alpha_{2}-\beta_{2}\right) q_{2} f\left(t_{1}\right)\right. \\
& \left.+\left(\alpha_{3}-\beta_{3}\right) q_{3} f\left(t_{1}\right)+\left(\alpha_{4}-\beta_{4}\right) q_{4} f\left(t_{0}\right)\right]
\end{aligned}
$$

$f(t)=\frac{1}{\sqrt{2 \pi} \sigma} e^{-\frac{1}{2}\left(\frac{t-\mu}{\sigma}\right)^{2}}$

$\alpha_{i}=$ fitness of conics in patch $i$

$\beta_{\mathrm{i}}=$ fitness of bents in patch $\mathrm{i}$

$\mathrm{q}_{\mathrm{i}}=$ probability organism develops in patch $\mathrm{i}$

$\sigma^{2}=$ variance in response thresholds

(The standard deviation in thresholds was set at 0.1 )

$$
\begin{aligned}
& Q=\left(\alpha_{1} q_{1}+\alpha_{2} q_{4}\right) \int_{t_{0}}^{\infty} f(x) d x \\
& +\left(\alpha_{1} q_{2}+\alpha_{2} q_{3}\right) \int_{t_{1}}^{\infty} f(x) d x \\
& +\left(\beta_{1} q_{1}+\beta_{2} q_{4}\right) \int_{-\infty}^{t_{0}} f(x) d x \\
& +\left(\beta_{1} q_{2}+\beta_{2} q_{3}\right) \int_{-\infty}^{t_{1}} f(x) d x .
\end{aligned}
$$

The $\mathrm{q}_{\mathrm{i}}$ are given by

$\mathrm{q}_{1}=\operatorname{Pr}($ no cue $\mid$ benign patch $) \operatorname{Pr}($ benign patch $)=F p$

$\mathrm{q}_{2}=\operatorname{Pr}($ cue $\mid$ benign patch) $\operatorname{Pr}($ benign patch $)=(1-\mathrm{F}) \mathrm{p}$

$\mathrm{q}_{3}=\operatorname{Pr}($ cue $\mid$ harsh patch $) \operatorname{Pr}($ harsh patch $)=\mathrm{G}(1-\mathrm{p})$

$\mathrm{q}_{4}=\operatorname{Pr}($ no cue $\mid$ harsh patch $) \operatorname{Pr}($ harsh patch $)=(1-G)(1-\mathrm{p})$.

The winning strategy is determined by solving for the mean response threshold $\mu$ when $\mathrm{S}=0$ (i.e. the equilibrium response threshold mean). The conditional strategy (C) is the winning strategy, if over $95 \%$ of the population has thresholds between $t_{1}$ and $t_{0}$ when the equilibrium response threshold has been reached. Stable mixed strategies are said to exist if, at equilibrium, more than one strategy occurs, but at a frequency of less than 0.95 . 
Figure 4 illustrates the results of the ET model in a form comparable to those of the ESS model (Figure 3). Notice the symmetry of the $\mathrm{U}$ or $\mathrm{D}$ area (conic or bent) area to the $\mathrm{C}$ (conditional) area. No such area exists in figure 3. In the $U$ or $D$ parameter space there is no one ESS but a mixture of possible winning strategies. In this parameter space the equilibrium mean (when $S=0$ ) is unstable, and therefore which strategy (U or D) becomes the winning strategy is expected to be random in a finite population.

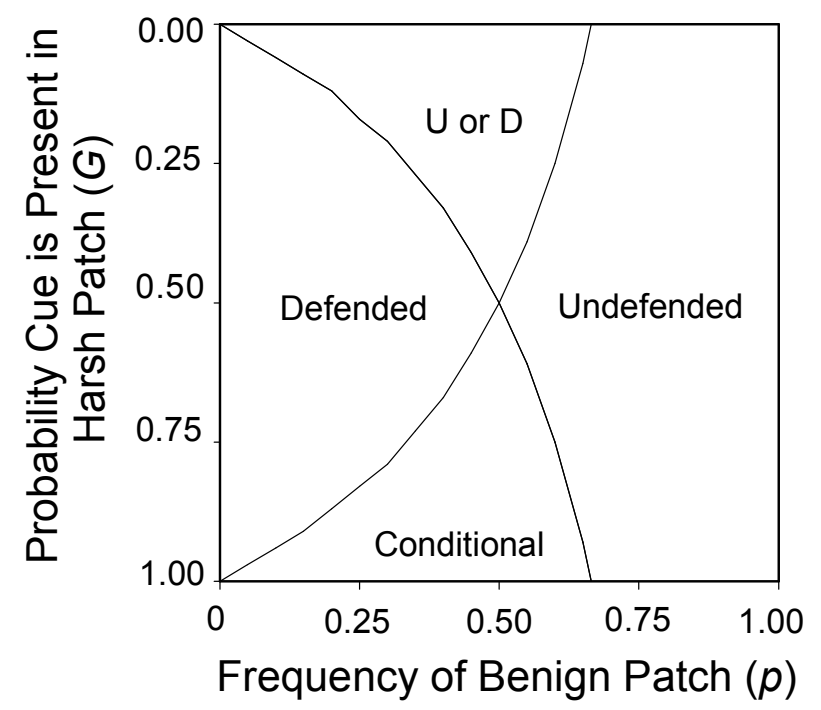

Figure 4: Parameter space for the Evolutionary Threshold Model

\subsection{Comparison of the ET and Strategic Models}

A difference in the predictions of the strategic and ET models is apparent. The strategic model predicts three areas of parameter space in which each of the conditional, defended and undefended strategies is the evolutionary stable strategy (ESS). (Shown in figure 3) In contrast, the ET model shows three areas of parameter space where the same three strategies exist at equilibrium; however, there is a fourth area where the outcome of selection is mixed.

The two interdisciplinary projects (biology and mathematics) involving two different analytical models -- and the differences in the predictions of the models - motivated a third project to test the ET model: a computer simulation that would use the same parameter space used to generate figures 3 and 4 .

\section{THE COMPUTER SCIENCE PERSPECTIVE}

\subsection{The $(\mu, \lambda)$-ES}

Twenty genes encode each barnacle's response threshold. Each gene is initialized randomly to a one or a zero. Summing the genes determines the barnacle's sensitivity (response threshold) to the snail's cue: with sensitivity ranging from zero to twenty.

The simulation uses a two-dimensional array to denote a section of the Gulf of California. Four zones divide the array into the four patches defined by the combinations of harsh or benign patches (snail or no snail) and cue present or not present.

A standard evolutionary computation loop simulates barnacle generations. The loop outline follows:

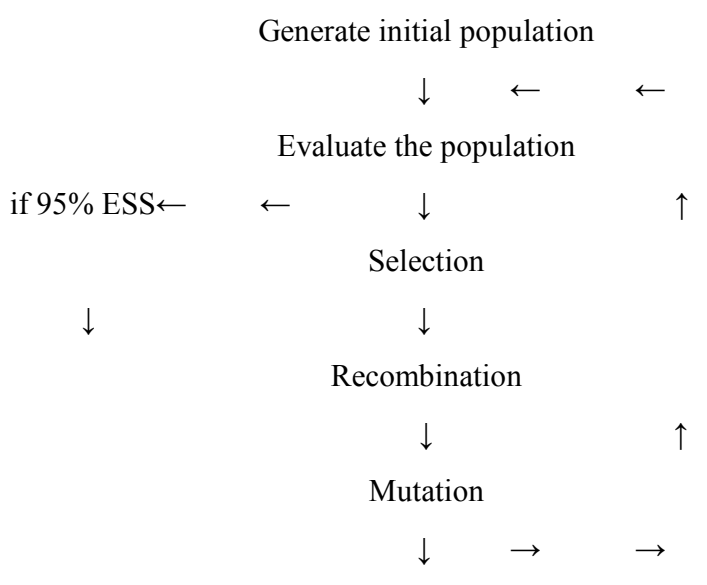

Pairs of barnacles are selected randomly in each patch as parents for the next generation. Recombination and mutation follow the evolution strategy, $(\mu, \lambda)$-ES, where $\mu$ individuals produce $\lambda$ offspring and the next generation is formed by choosing $\mu$ of the $\lambda$ offspring for the next generation [14]. Each barnacle $(\mathrm{x}, \sigma)$ consists of twenty genes $(\mathrm{x})$ and twenty standard deviations $(\sigma)-$ in a one-to-one correspondence, as described by $(\mu, \lambda)$-ES. Recombination averages the corresponding genes and standard deviations of each parent barnacle.

Mutation [14] for each offspring ( $\mathrm{x}, \sigma)$, if $\mathrm{x}$ is the sequence of alleles and $\sigma$ is the sequence of standard deviations, produces an offspring $\left(\mathrm{x}^{\prime}, \sigma^{\prime}\right)$, where

$\sigma^{\prime}=\sigma \cdot \mathrm{e}^{\mathrm{N}(0, \Delta \sigma)} \quad$ and

$x^{\prime}=x+N\left(0, \sigma^{\prime}\right)$

\subsection{Parameter Decisions}

The simulation adheres to $(\mu, \lambda)$-ES, using 0.005 for the initial standard deviation sequence, by extrapolating from the standard deviation used in the ET model detailed in section 1.2. The standard deviation used in the mathematical derivations in section 1.2 is 0.1 for a population with a mean that bisects a normally distributed range of possible sensitivities, $-\infty$ to $+\infty$. The simulation's initial population has a mean of ten for barnacle sensitivities, where the sensitivities range from zero to twenty. The evolutionary algorithm must relate the twenty standard deviations to the twenty alleles, where each mean is 0.5 .

The ET model does not provide cutoff points for $t_{0}$ and $t_{1}$. Experimentation with all combinations of possible $t_{0}$ and $t_{1}$ values as parameters for the simulation establishes choices of 5 and 7 . 
The ET model gives no assistance, because ET sensitivity varies from $-\infty$ to $+\infty$.

Experimentation leads to a $\Delta \sigma$ value of .0000001 , allowing the populations of each data point to reach stability. Stability indicates one strategy is adopted by $95 \%$ or more of the population or two strategies exist, such that repeated generations do not advance the establishment of one winning strategy.

Finally, the program repeats its experiments multiple times, counting the strategy winners for each data point and declaring the final data point choice as the most frequent result.

\subsection{Results}

The new prediction graph follows, where the axes retain the same labels as figures 3 and 4 . The vertical axis represents the probability that the cue is present in the harsh patch $(G)$; the horizontal axis, the frequency of the benign patch (p). The label $\mathrm{D}$ indicates that the defended strategy is held by $95 \%$ of the population; $\mathrm{U}$, undefended; $\mathrm{C}$, conditional. The borders between winning strategies are blurred, as expected. The populations of the data points in the border areas share two strategies: < indicates a mixture of strategies $\mathrm{D}$ and $\mathrm{C} ;>>\mathrm{U}$ and $\mathrm{C}$.

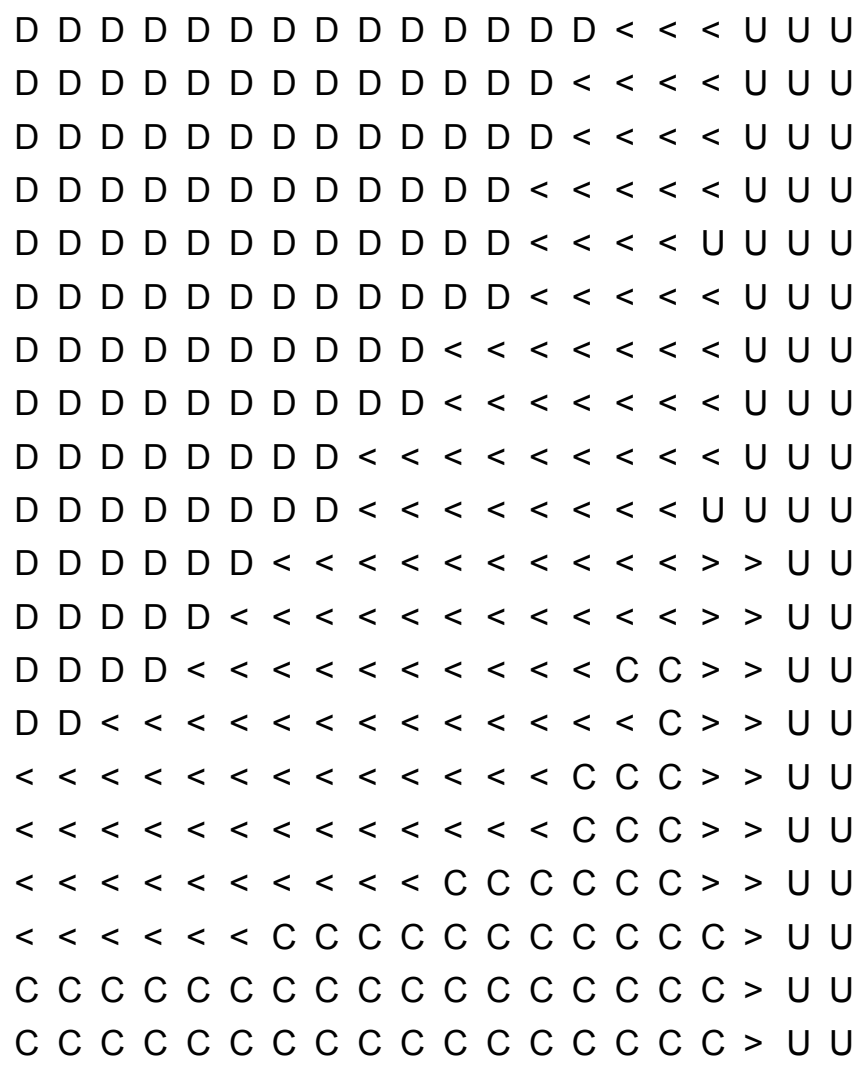

Figure 5: Data points for the evolutionary computation algorithm $(\mu, \lambda)$-ES, established for the purpose of comparison with the data points in figures 3 and 4.

\subsection{Comparison to the Analytical Models}

The results of the evolutionary computation simulation appear to support the strategic model (figure 3). The three distinct D, U and
$\mathrm{C}$ areas retain their respective regions with borders among the regions containing the expected mixtures of strategies. There is no "U or D" area, as in the environmental threshold model (figure 4).

However, superimposing the "U or D" area (labeled as such in figure 4) data points on the data points above in figure 5 and checking the corresponding statistics reveals that the frequencies of times that the defended strategy wins $95 \%$ of the time is not close to the total number of times that the simulation is run. That is, the defended strategy is the most frequent winner for each data point in the "U or D" area - but approximately $25 \%$ of the time the undefended strategy is adopted by $95 \%$ or more of the population. By contrast, almost all of the undefended data points in figure 5 correspond to statistics, which indicate that the undefended strategy is adopted by at least $95 \%$ of the population in $95 \%$ or more of the repetitions of the simulation. Therefore, the evolutionary computation simulation indicates similarities between the parameter spaces of both of the two analytical models - similarities that were not readily apparent in figures 3 and 4.

\section{CONCLUSIONS}

The project's goal lay in determining if evolutionary computation algorithms could support one of two competing models for evolutionary stable strategies, applied to a specific biological problem. The project achieves its goal through the results pictured in figure 5. Moreover, the results suggest that each group of authors might return to the research, which produced the results shown in figures 3 and 4, to examine the parameter space labeled "U or D" in the respective analytical model and the parameter space generated by the current simulation or its future versions.

\section{FUTURE WORK}

Funding from the National Science Foundation will allow all three scientists to continue work, during the next two summers. A selected list of circumstances and possibilities follows:

- The research is accessible to undergraduates. Students originally developed their own algorithms [1] (based upon concepts they learned in introductory genetics classes) to model the evolution of snail/barnacle colonies. Student results motivated the described research project that uses, as an alternative, a standard algorithm from the literature of evolutionary computation. Undergraduates will assist in the next phases of research.

- One important question to answer by computer simulation is what happens to the variance in sensitivity once the population reaches its equilibrium. The simulation should be extended to take into account variance in exposures to the environmental cue, as well as variance in response to the cue.

- Work that explores prediction graphs (with the same parameters as those in figures 3 and 4) -- where competitive interactions among barnacles are allowed -- is included in the same paper [5] as the ET model discussed in this paper. Modifying the existing simulation to accommodate competitive interactions is a natural next step.

- The described simulation requires hours of CPU time to complete several runs. Investigating parallel computation as a means to continue the research would be practical. 


\section{ACKNOWLEDGMENTS}

This study was supported by grants from the DePauw University Faculty Development Program and the National Science Foundation grants DEB-9317924 and DEB-0223089.

\section{REFERENCES}

[1] Briney, K. and Karpinski T. An interdisciplinary investigation of the Evolution and maintenance of conditional strategies in Chthamalus anisopoma, using genetic programming and a quantitative genetic model. Proceedings of the 2003 Genetic and Evolutionary Computation Conference (GECCO) Workshops Program, 2003, Chicago.

[2] Hazel, W. N., Smock, R., and Johnson, M. D. A polygenic model for the maintenance and evolution of conditional strategies. Proceedings of the Royal Society, Series B. 242, 1990, 181-187.

[3] Hazel, W. N. and Smock, R. Modeling selection on conditional strategies in stochastic environments. Pages 147154 in J. Yoshimura and C. Clark (Eds.), Lecture Notes in Biomathematics, Springer-Verlag, 1993.

[4] Hazel, W. N. and Smock, R. Inheritance and the conditional strategy revisited. Journal of Theoretical Biology, 204 (2000), 307-309.

[5] Hazel, W. N., Smock, R., and Lively, C. M. The ecological genetics of conditional strategies. The American Naturalist, 163, 6 (June 2004).

[6] Levins, R. Evolution in Changing Environments. Princeton University Press. Princeton, N.J., 1968.
[7] Lively, C. M. Predator-induced shell dimorphism in the acorn barnacle, Chthamalus anisopoma. Evolution, 40 (1986a), 232-242.

[8] Lively, C. M. Competition, comparative life histories, and the maintenance of shell dimorphism in a barnacle. Ecology, 67 (1986b), 858-864.

[9] Lively, C. M. Competition, comparative life histories, and the maintenance of shell dimorphism in a barnacle. Ecology, 67 (1986c), 858-864.

[10] Lively, C. M. Developmental strategies in spatially variable environments: barnacle shell dimorphism and strategic models of selection. R. Tollrian and C. D. Harvell, editors. The Ecology and Evolution of Inducible Defenses. Princeton University Press, Princeton, N.J., 1999, 245-258.

[11] Lively, C. M., Hazel, W. N., Schellenberger, M. J., and Michelson, K. S. Predator-induced defense: variation for inducibility in an intertidal barnacle. Ecology, 81 (2000), 1240-1247.

[12] Lloyd, D. G. Variation strategies of plants in heterogeneous environments. Biological Journal of Linnean Society, 27 (1984), 357-385.

[13] Maynard Smith . J. Evolution and the Theory of Games. Cambridge University Press, Oxford, 1982.

[14] Michalewicz, Z. Genetic Algorithms + Data Structures $=$ Evolution Programs. Springer-Verlag, Berlin, Germany, 1996.

[15] Moran, N. A. The evolutionary maintenance of alternative phenotypes. The American Naturalist, 139 (1992), 971-979 